\title{
Successful resection of the recurrence of a cavernous malformation of the optic chiasm
}

\author{
Martin Scholz \\ Albrecht Harders \\ Sebastian Lücke \\ loannis Pechlivanis \\ Martin Engelhardt \\ Kirsten Schmieder \\ Department of Neurosurgery, \\ Ruhr-University Bochum, \\ Knappschaftskrankenhaus, Germany
}

\begin{abstract}
The case of a 33-year-old female who suffered from a recurrence of an intrachiasmatic cavernous malformation is presented. She had already undergone surgery in 1991 and 2001 and was admitted to our hospital with reduced vision in the right eye. After MRI, and diagnosis of recurrence of the cavernoma, a neurosurgical operation was performed using the pterional approach. The intraoperative situation was documented with micro photographs. The postoperative course was uneventful. The female described a minimal improvement of her vision. No postoperative complications were observed. To our knowledge, microsurgically complete extirpation of a recurrence of an intrachiasmatic cavernoma has not yet been reported in the literature.
\end{abstract}

Keywords: optic chiasm, pterional approach, cavernous malformation

\section{Introduction}

An intracranial cavernous malformation can develop in several locations of the central nervous system. A cavernous malformation can be found in the cerebrum, the cerebellum, or the spinal cord. Sometimes they are associated with other venous malformations. Cavernous malformations of the chiasm are rarely reported. Lehner and colleagues (2006) have provided a recent literature review. To our knowledge, microsurgical extirpation of a recurrence of an intrachiasmatic cavernoma has not been reported previously.

\section{Materials and methods Case description}

A 33-year-old female was admitted to our department as an emergency case. Her vision had decreased progressively for two weeks, and was characterized by a worsening right homonymous hemianopsia, which had been stable for many years. She also reported headache and nausea. Her medical history included surgery for an intrachiasmatic cavernoma in 1991 and 2001, in another neurosurgical university department. The cavernoma was partially resected and followed up with magnetic resonance imaging (MRI) studies. A fusiform enlargement of the internal carotid artery was diagnosed in 2006 by cerebral angiography. Neurosurgical treatment of this finding was not thought to be necessary. The complaints had begun with flu three weeks before, which had improved during the last few days. She complained of biparietal headache and the feeling of pressure behind both eyeballs, accentuated on the right side.

Neurologic examination showed a female in normal physical condition. Right visual acuity was 2/20 and left was 20/20.

The pupils measured $3 \mathrm{~mm}$, were reactive to light, with no relative afferent pupillary defect. Conjunctival injections were seen bilaterally and could not be explained. 
Right homonymous hemianopsia was found. The preoperative visual field can be seen in Figure 1. During neurological examination, motor strength was normal. Sensory testing showed no deficits. Reflexes were normal. Cerebellar testing showed regular function. Cranial nerve exam was normal.

\section{Neuroradiologic findings}

MRI revealed a 1.5-cm hypointense lesion on T2-weighted images inside the optic chiasm in the suprasellar region (Figure 2). T1-weighted noncontrast-enhanced images showed a hyperintense intra-axial lesion intrinsic to the optic chiasm, suggesting a cavernoma.

A

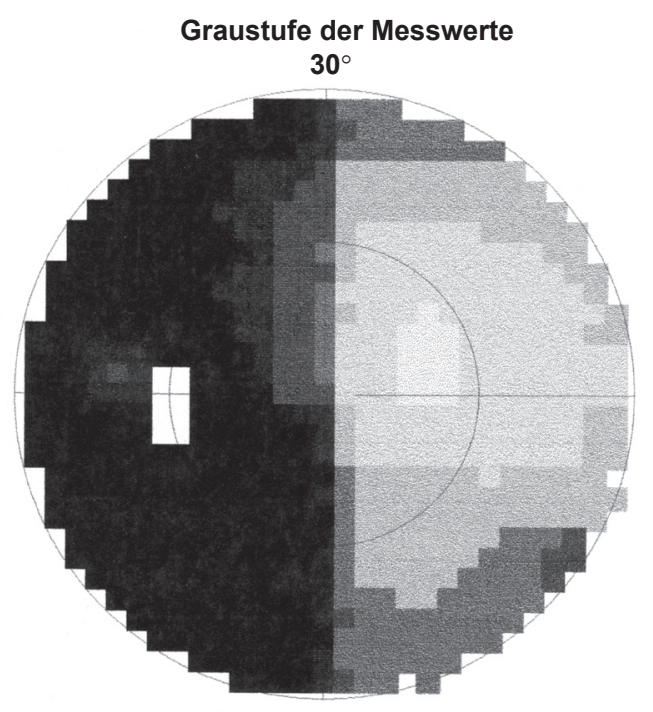

B

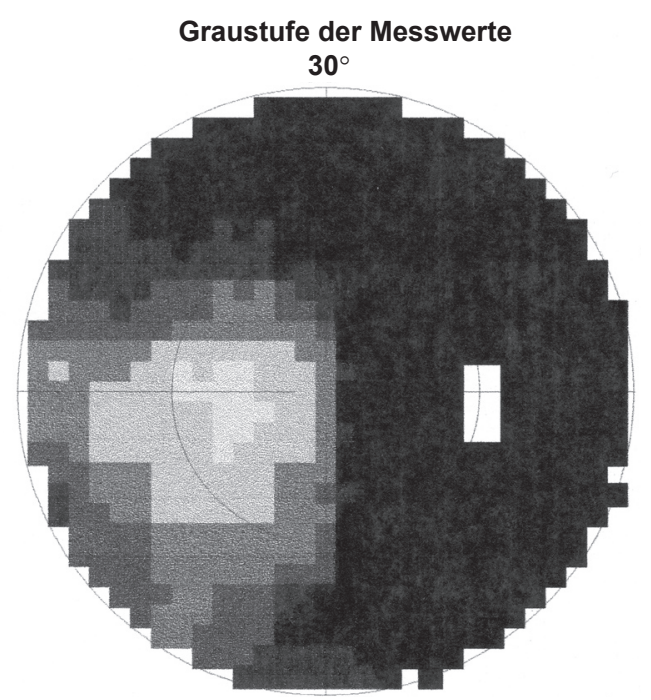

Figure I The preoperative visual field. A) Left eye. B) Right eye.
After the administration of gadolinium the lesion showed heterogenous enhancement (Figure 3).

Surgery was performed on November 13, 2007 , using a right-hand pterional approach and an osteoplastic temporoparietal trepanation. Figures $4 \mathrm{~A}$ and $4 \mathrm{~B}$ give an impression of the intraoperative field. After microsurgical separation of adherences between the cerebral cortex and dura, the transsylvian approach was used, and basal cisterns were opened. The optic chiasm was clearly visible as well as the carotid artery, which was enlarged fusiform as described in the previous angiography. Lamina terminalis was opened, then the chiasm was opened at the thinnest part, in order to resect the cavernomas completely under the microscope. After establishing control of an absolutely dry surgical field (Figure 5) the head was closed in layers with watertight sutures of the dura, reimplantation of the bone flap, insertion of a drain and stitches of the galea and skin.

\section{Results}

The postoperative course was uneventful. Postoperative computed tomography (CT) demonstrated a regular postoperative state after pterional trepanation and surgical

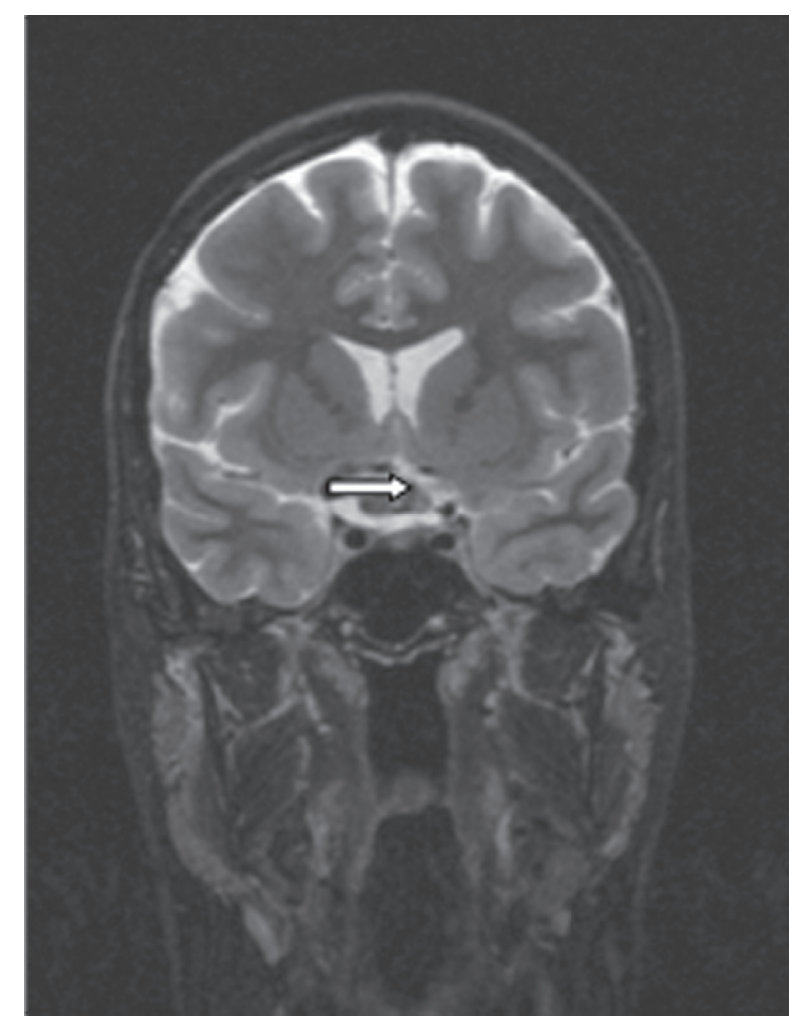

Figure $2 \mathrm{MRI}$, coronal section, T2-weighted image: the enlargement of the optical chiasm can easily be seen. 


\section{A}

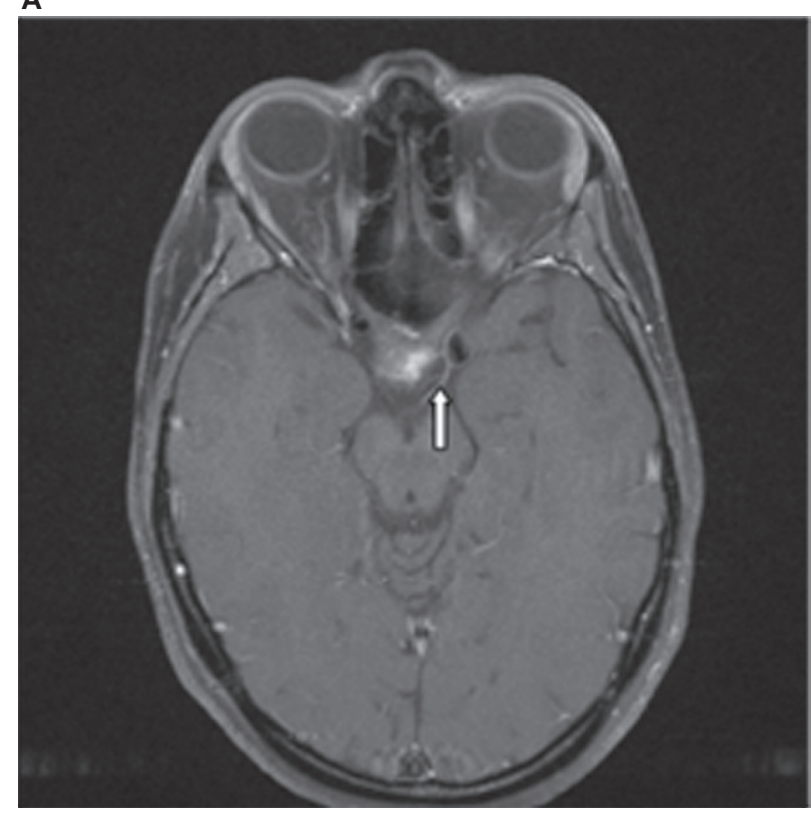

B

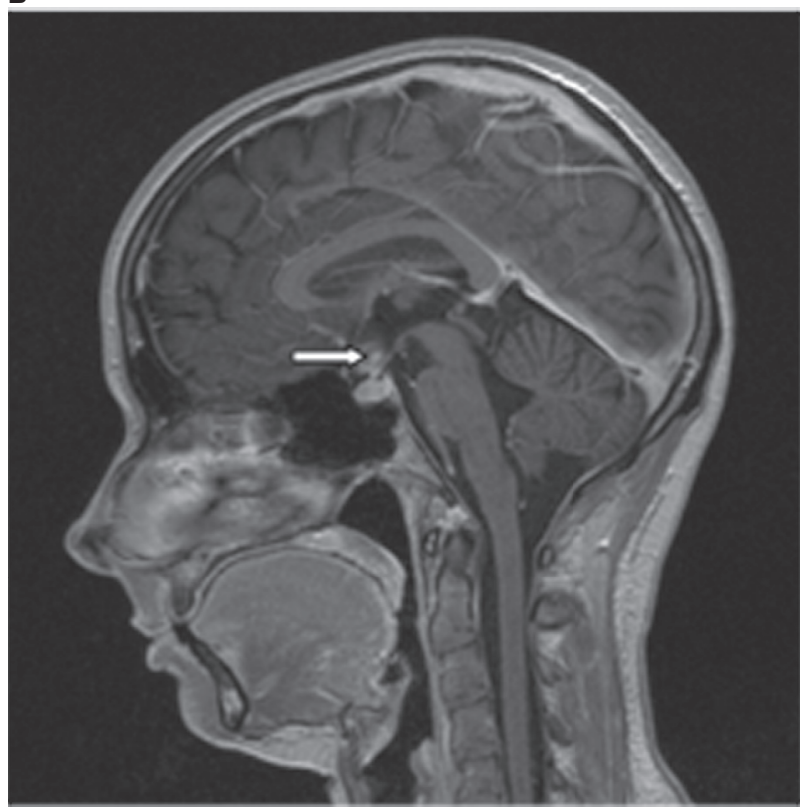

Figure 3 A) MRI, axial section, TI -weighted images after administration of gadolinium: the irregular contrast enhancement (arrow) can be seen. B) MRI, sagittal section, TI-weighted image after administration of gadolinium: the enlarged chiasma suprasellar above the pituitary gland can be seen.

extirpation of a cavernous malformation without midline shift or intracranial hemorrhage but with some intracranial air inclusions.

After one day in the intensive care unit the patient was transferred to the normal ward. She demonstrates no additional neurological deficits. Early ophthalmologic examination revealed minimal improvement of vision in the right eye. The visual field was unchanged. Histological examination
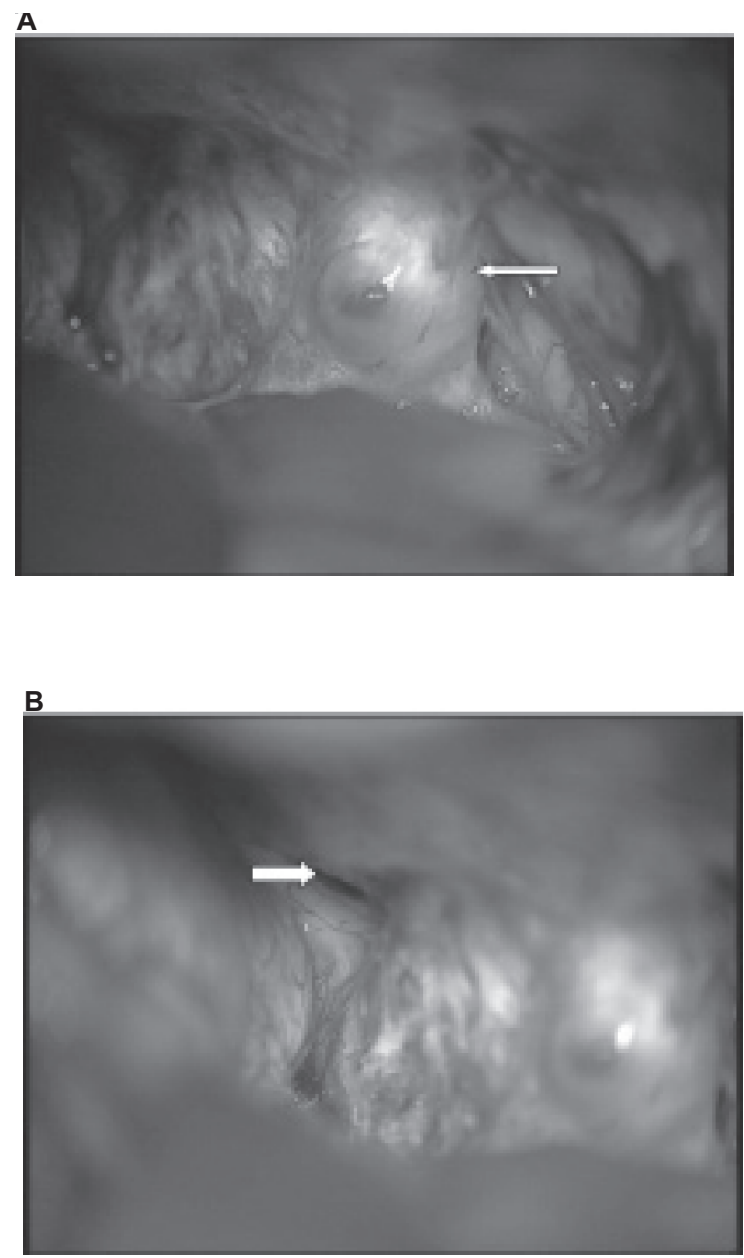

Figure 4A) Intraoperative photograph after pterional approach from the right-hand side and splitting of the sylvian fissure: blue scarring in the region of lamina terminalis can be seen together with a bulging and thinning of the right-hand optic nerve (arrow). B) Intraoperative photograph after pterional approach from the right-hand side: the focused contralateral yellow optic nerve (arrow) seems uninvolved by the lesion with normal vascularization.

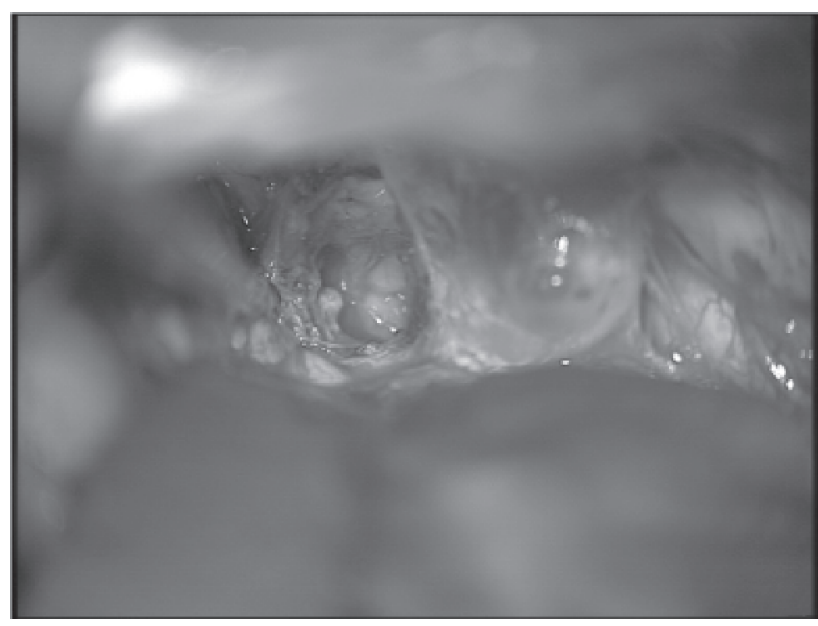

Figure 5 Intraoperative photograph after pterional approach from the right-hand side and resection of the cavernoma. The suction tube touches the beginning of the left optic nerve. One can look through the right-hand optic nerve, because it is extremely thin. 
revealed a typical cavernous malformation. Postoperative MRI (Figures 6A-C) demonstrated no residual cavernoma but some hemosiderin deposits near to the chiasm.

\section{Discussion}

In their meticulous review of the literature, Lehner and colleagues (2006) found 42 previously reported patients with vascular malformations within optic nerves, chiasm, or optic tracts, 30 of them being cavernous hemangiomas. The optic chiasma was involved in 38 patients $(90.5 \%)$ and a total excision of the tumor was performed in 21 cases. Different types of cavernomas have been described previously (Ojemann et al 1993; Aiba et al 1995; Kondziolka et al 1995; Bertalanffy et al 2002).

Nearly 40 reports in total deal with histologically verified cavernomas involving one or more parts of the intracranial optic pathway (optic nerve, chiasm, and optic tract) (Shaikh et al 2001; Ozer et al 2007; Santos-Ditto et al 2007). Thus several reports on intrachiasmatic cavernomas have so far been published. Nevertheless only a few discuss the surgical treatment of such lesions. Complete resection of intrachiasmatic lesions, rather than biopsy, is seldom reported. Hempelmann and colleagues (2007) presented a case of the first complete resection of a cavernous hemangioma, and all three parts of the anterior intracranial optic pathway (optic neve, chiasm, and tract). Paladino and colleagues (2001) reported a case of a cavernoma in the chiasm, which was operated on via an eyebrow incision. This small frontolateral trepanation is a fast forward procedure, does not need retraction of temporal muscle and is a good solution in case of a primary cavernoma in the chiasmatic region. In the case presented, we used the old pterional trepanation and skin incision behind the hairline because this approach gives more space and allows a better overview, especially if the patient has undergone surgery previously. We present detailed intraoperative photographs to clearly illustrate our surgical technique.

Clinical findings are sudden apoplectiform visual disturbances such as those reported in our case. During this "chiasmal apoplexy" (Maitland et al 1982) nearly all patients suffer from headache. Several cases of recurrent bleeding of cavernomas of the chiasm or the optic nerve have been reported (Hassler et al 1989; Steinberg et al 1990). These known recurrences indicate that a complete operative removal of the lesion should be attempted.

Microsurgical extirpation is also possible in case of a recurrence but the anatomical situation is much more complicated by adhesions and intracranial scaring.

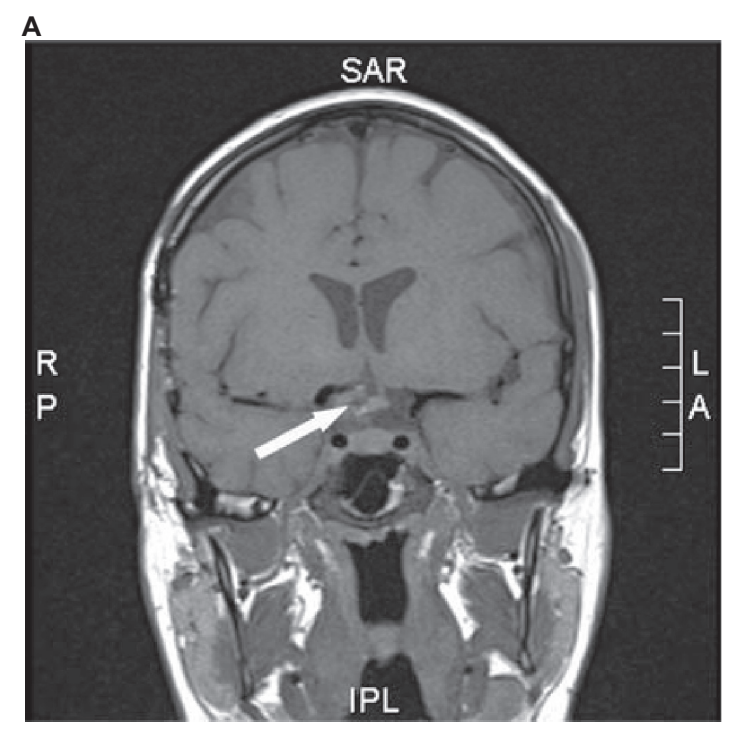

B

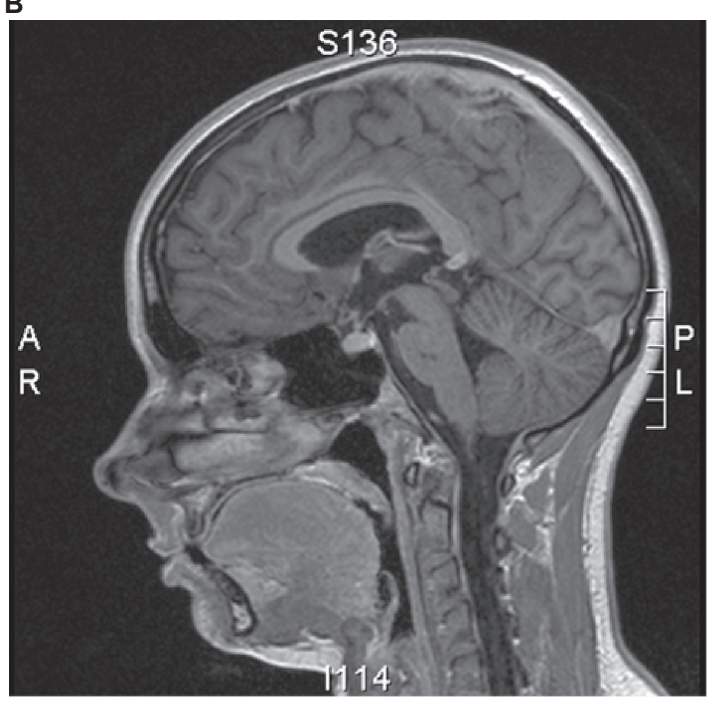

C

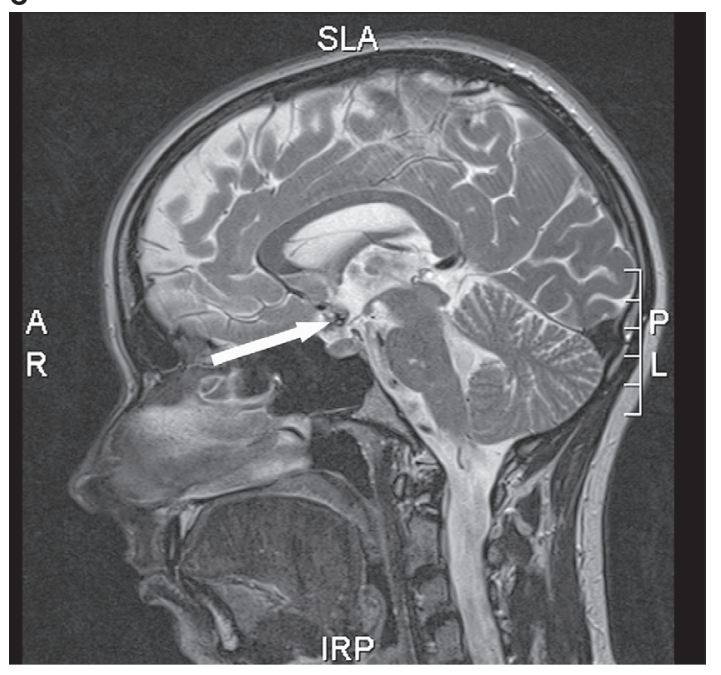

Figure 6 Postoperative MRI. A) MRI, coronal section, TI-weighted images: a postoperative defect inside the chiasm is visible. B) MRI, sagittal section, $\mathrm{TI}$-weighted images after administration of gadolinium: no residual cavernoma is visible. C) MRI, sagittal section, $\mathrm{T} 2$-weighted images revealing complete resection of the cavernoma with minimal hemosiderin residuals. 
Recurrences of cavernomas can be explained by residual, forgotten or left behind pieces of the cavernoma during a previous intervention, which grow as a result of microhemorrhages inside the venous network of these lesions.

In the case presented, the visual acuity is very asymmetric. It is possible that the fusiform enlargement of the internal carotid artery was partially responsible for the optic atrophy of the right eye.

After complete resection, vision improves in most cases. Therefore complete resection should be the aim of the operation when possible.

\section{Disclosure}

The authors report no conflicts of interest in this work.

\section{References}

Aiba T, Tanaka R, Koike T, et al. 1995. Natural history of intracranial cavernous malformations. J Neurosurg, 83:56-9.

Arrue P, Thorn-Kany M, Vally P, et al. 1999. Cavernous hemangioma of the intracranial optic pathways: CT and MRI. J Comput Assist Tomogr, 23:357-61.

Awad I, Jabbour P. 2006. Cerebral cavernous malformations and epilepsy. Neurosurg Focus, 21:e7.

Bertalanffy H, Benes L, Miyazawa T, et al. 2002. Cerebral cavernomas in the adult. Review of the literature and analysis of 72 surgically treated patients. Neurosurg Rev, 25(1-2):1-53; discussion; 54-5.

Hassler W, Zentner J, Wilhelm H. 1989. Cavernous angiomas of the anterior visual pathways. J Clin Neuroophthalmol, 9:160-4.
Hempelmann RG, Mater E, Schröder F, et al. 2007. Complete resection of a cavernous haemangioma of the optic nerve, the chiasm, and the optic tract. Acta Neurochir (Wien), 149:699-703; discussion, 703.

Kondziolka D, Lunsford LD, Kestle JR. 1995. The natural history of cerebral cavernous malformations. J Neurosurg, 83:820-4.

Lehner M, Fellner FA, Wurm G. 2006. Cavernous haemangiomas of the anterior visual pathways. Short review on occasion of an exceptional case. Acta Neurochir, 148:571-8.

Maitland CG, Aminoff MJ, Kennard C, et al. 1982. Evoked potentials in the evaluation of visual field defects due to chiasmal or retrochiasmal lesions. Neurology, 32:986-91.

Ojemann RG, Crowell RM, Ogilvy CS. 1993. Management of cranial and spinal cavernous angiomas (honored guest lecture). Clin Neurosurg, 40:98-123.

Ozer E, Kalemci O, Yücesoy K, et al. 2007. Optochiasmatic cavernous angioma: unexpected diagnosis. Case report. Neurol Med Chir (Tokyo), 47:128-31.

Paladino J, Rotim K, Pirker N, et al. 2001. Minimally invasive treatment of cavernous angioma of the optic chiasm: case report. Minim Invasive Neurosurg, 44:114-6.

Santos-Ditto RA, Santos-Franco JA, Pinos-Gavilanes MW. 2007. [Cavernous angioma of the second cranial nerve and chiasmatic apoplexy]. Neurocirugia (Astur), 18:47-51.

Schiefer U, Isbert M, Mikolaschek E, et al. 2004. Distribution of scotoma pattern related to chiasmal lesions with special reference to anterior junction syndrome. Graefe's Arch Clin Exp Ophthalmol, 242:468-77.

Shaikh A, Benjamin L, Kerr R. 2001. Chiasmal cavernous angioma. A rare case of progressive visual loss. Eye, 16:655-7.

Shibuya M, Baskaya MK, Saito K, et al. 1995. Cavernous malformations of the optic chiasma. Acta Neurochir (Wien), 136:29-36.

Steinberg GK, Marks MP, Shuer LM, et al. 1990. Occult vascular malformations of the optic chiasm: magnetic resonance imaging diagnosis and surgical laser resection. Neurosurgery, 27:466-70.

Tien R, Dillon WP. 1989. MR imaging of cavernous hemangioma of the optic chiasm. J Comput Assist Tomogr, 13:1087-8. 
